\title{
Review of: "Generation mechanism and prediction of an observed extreme rogue wave"
}

\author{
Francesco Fedele ${ }^{1}$ \\ 1 Georgia Institute of Technology
}

Potential competing interests: The author(s) declared that no potential competing interests exist.

The authors develop an empirical model based on data regression of some relevance. However, their analysis and predictions are somewhat flawed because:

1. data utilized does not represent an ensemble of sea states with similar statistical characteristics. In this case, the principle statistical parameters comprise $r$, variance, skewness and kurtosis. These vary between sea states. Scaling the wave height and crest data with $H_{s}(=4 \sigma$ ?) eliminates the variability of variances but not others.

2. Further, some data likely represent mixed seas situations, e.g. swell + wind seas or two different wind seas. In general, there are no rigorous models for describing the distributions of wave heights or crests in such seas.

3. Combining records gathered from mixed sea states and/or those characterized with different $r$, skewness and kurtosis in a composite as if the resulting ensemble represented the outcomes from the same random process is not appropriate. What is needed is an ensemble comprised of buoy records characterized with adequately similar statistical characteristics, say, within less than a maximum of $+/$ $10 \%$ variation, excluding those cases clearly recognized as mixed sea state situations. The latter necessitates spectral analysis.

4. If such filtering is done based on the similarity of skewness and kurtosis but not $r$, then a regression of the rogue probability $P\left(H>2.2 H_{s}\right)$ based on wave data characterized by variable $r$ in the same population (as in the authors' case) can be carried out as follows: describe $r$ in a number of discrete intervals or bins. Each bin contains $N_{j}$ sea states and it is centered at $r_{j}$ with width $\Delta r$. Drawing on Fedele (2016), the rogue probability is

$$
P\left(H>2.2 H_{s} \mid r=r_{j}\right)=\int P(H>2.2 H s \mid r=x) p\left(r=x \mid r=r_{j}\right) d x
$$

where $p(r)$ is the probability density function of $r$ associated to the bin and $p\left(r=x \mid r=r_{j}\right) d x$ is the number of sea states with crest-trough correlation $r$ in $[x, x+d x]$. In discrete form

$$
P\left(H>2.2 H_{s} \mid r=r_{j}\right)=\sum_{k=1, N_{j} P\left(H>2.2 H_{s} \mid r=r_{j}\right)}^{\frac{N_{j k}}{N_{j}}},
$$

where $N_{j k} \sim p\left(r=x_{k} \mid r=r_{j}\right) \Delta x$ is the number of sea states with crest-trough correlation $r$ in $\left[x_{k}, x_{k}+\Delta x\right]$, 
and we subdivide the bin width in $M$ parts $\left(\Delta x=\Delta_{r} / M\right)$. Given a sea state, the conditional rogue probability $P\left(H>2.2 H s \mid r=r_{j}\right) \sim R_{j k} / M_{j k}$ follows from the data. Here, $R_{j k}$ is the number of waves of the $k^{\text {th }}$ sea state whose height exceeds $2.2 H_{s}$, and $M_{j k}$ is the total number of waves observed in that sea state. So,

$$
P\left(H>2.2 H_{s} \mid r=r_{j}\right)=\sum_{k=1, N_{j}} \frac{R_{j k}}{M_{j k}} \frac{N_{j k}}{N_{j}}
$$

It appears that the authors have instead mixed all waves from different sea states as part of the same population and estimated the rogue probability as

$$
P\left(H>2.2 H_{s} \mid r=r_{j}\right)=\frac{\sum_{k=1, N_{j}} R_{j k}}{\sum_{k=1, N_{j} M_{j k}}}
$$

by assigning the same $r_{j}$ to all the sea states observed within a bin. This is an issue of some concern here. A simple formula for the rogue probability can also be derived by Taylor-expanding the PDF $p(x)$ up to second order obtaining (Fedele 2016)

$$
P\left(H>2.2 H_{s} \mid r=r_{j}\right)=P\left(H>2.2 H_{s} \mid r=r_{j}\right)+\frac{\frac{1}{2} \frac{\partial^{2} P\left(H>2.2 H_{s} \mid r=r_{j}\right)}{\partial r^{2}}}{\sigma_{r}^{2}}
$$

where $r_{j}$ and $\sigma_{r}^{2}$ are the average value and variance of the parameter $r$ observed within the bin interval. This formula can be used to compare their predictions with theoretical models as described below.

5. buoy data is not the best alternative for developing models, especially for wave crests. Further, wave breaking effects are not mentioned at all whereas drooping of the probability observed toward the extremes are indicative of such effects.

6. what does second-order model mean in their plots and analysis? Why is the steepness $\varepsilon$ defined as $k_{p} \sigma$ ? That underestimates the second-order predictions particularly for narrow-band waves (largest $r$ values in their data). The correct choice here is to set $\varepsilon=\sigma\left(w_{m}\right)^{2} / g=\sigma k_{m}$, with $k_{m}$ the mean wavenumber. For example, in deep waters $k_{m}=w_{m}^{2} / g$ with $w_{m}=m_{1} / m_{0}$ the mean frequency and $m_{j}$ the spectral moments of the frequency spectrum.

7. The authors do not compare their regressions with available theoretical models in the literature. They mention about the Naess (1985) and Tayfun (1990) models, but they don't compare their results with theoretical predictions. Furthermore, the authors are unaware that Boccotti $(1982,1983,1985,2000)$ originally introduced the cross-correlation parameter $\psi^{*}$ (similar to $r$ ) that naturally followed from its asymptotic analytical solution of the distribution of large wave heights in Gaussian seas of any spectral bandwidths. The Naess model is just an approximation of the wave height distribution for narrow-band waves (see letter to the editor by Boccotti (1986) in reply to Naess' (1985) work). The correct asymptotic model for wave heights in the limit of narrowband waves has been derived by Tayfun (1990) and reported below 
$P_{T}(y \mid r)=P\left(H>y H_{s} \mid r\right)=\sqrt{\frac{1+r}{2 r}}\left(1+\frac{1-r^{2}}{64 r y^{2}}\right) \exp \left(-\frac{4 y^{2}}{1+r}\right)$

Is the Tayfun model the second order model used by the authors in their plots and analysis?

Recently, Alkhalidi \& Tayfun (2013) have generalized the Boccotti model to nonlinear waves and have shown that it is the best model that predicts wave heights, including rogue waves.

8. Below I compared their regression against the Tayfun (1990) model and its extension to account for the variability of $r\left(\sigma_{-} r=0.25\right)$, leading to the expression

$$
P_{T}\left(H>\left.2.2 H_{S}\right|^{r}\right)=P_{T}(y=2.2 \mid r=r)+\frac{1}{2}\left(\frac{\partial^{2} P(y=2.2 \mid r)}{\partial r^{2}}\right)_{r=r}^{-\sigma_{r}^{2}}
$$

where the expression of the second derivative of $P_{T}$ with respect to $r$ is given by

$$
\frac{\partial^{2} P_{T}}{\partial r^{2}}=P_{T}(y \mid r) \frac{B_{1}+B_{2}}{4 r^{2}(r+1)^{4}\left(r^{2}-64 r y^{2}-1\right)}
$$

and

$$
\begin{gathered}
B_{1}=3 r^{4}\left(704 y^{4}-80 y^{2}-3\right)-2 r^{3}\left(2048 y^{6}-1536 y^{4}+312 y^{2}+21\right) \\
B_{2}=24 r^{2}\left(40 y^{4}-22 y^{2}-3\right)-18 r\left(8 y^{2}+3\right)-15
\end{gathered}
$$

The authors should compare their regression against the Tayfun and Boccotti models to validate their analysis. 


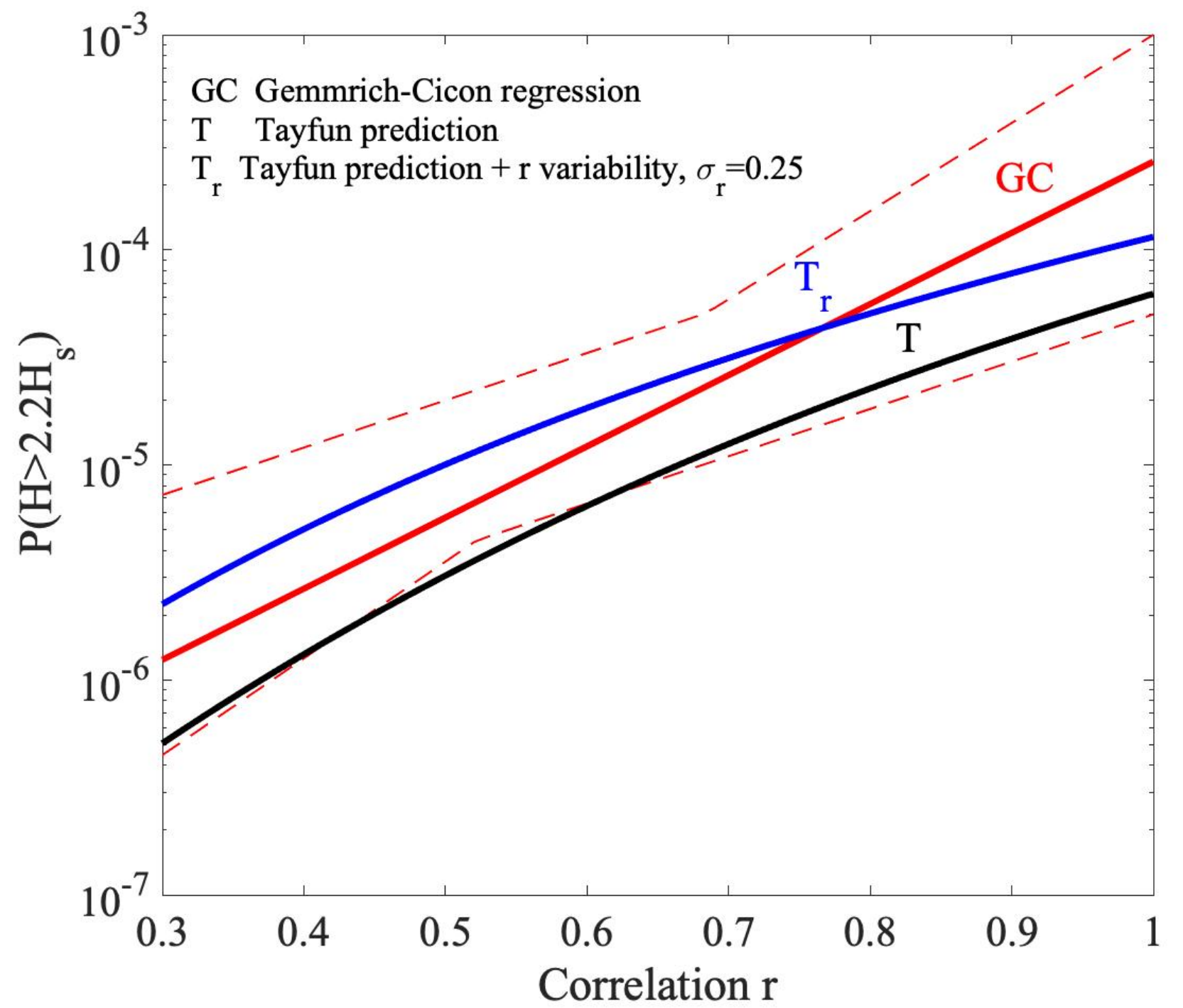

\section{REFERENCES}

Alkhalidi, M. A. \& Tayfun (2013), M. A. Generalized Boccotti distribution for nonlinear wave heights. Ocean Engineering 74, 101-106 (2013)

Boccotti, P., 1981. On the highest waves in a stationary Gaussian process. Atti Accademia Ligure di Scienze e Lettere 38, 271-302.

Boccotti, P., 1982. On ocean waves with high crests. Meccanica 17, 1

Boccotti 1982, Letter to the editor, Ocean Engineering, Volume 13, Issue 4, 1986, Pages 417-418

Boccotti, P. Wave Mechanics for Ocean Engineering (Elsevier Sciences, Oxford, 2000)

Fedele, F., Lugni, C. \& Chawla, A. The sinking of the El Faro: predicting real world rogue waves during Hurricane Joaquin. Scientific Reports 7, 11188 (2017).

Fedele, F. (2016). Are rogue waves really unexpected?. Journal of Physical Oceanography, 46(5), 14951508.

Tayfun, M. A. (1990). Distribution of large wave heights. Journal of waterway, port, coastal, and ocean 
engineering, 116(6), 686-707.

Tayfun, M. A. \& Fedele, F. Wave-height distributions and nonlinear effects. Ocean Engineering 34, 16311649 , 